\title{
Efecto de ácidos hidroxicarboxílicos en la biorregulación del estrés causado por herbicidas en el cultivo de tomate
}

\section{Effect of hidroxy-carboxylic acids on the bio-regulation of stress caused by herbicides in tomato crops}

JOREE A. RODRÍGUEZ1

GUIDO PLAZA ${ }^{2,3}$

Establecimiento de plantas

de tomate.

Foto: Jorge A. Rodríguez

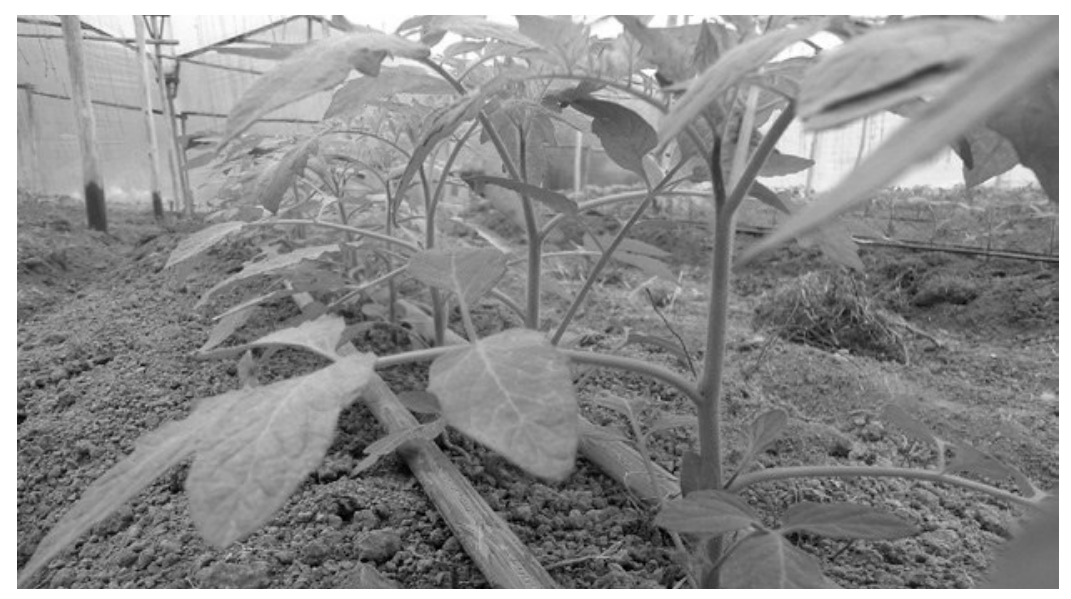

\section{RESUMEN}

La pérdida de selectividad de los herbicidas es un riesgo permanente y relevante en cultivos de alto valor; motivo por el cual es importante contar con alternativas que permitan mitigar su efecto en la fisiología del estrés en estos cultivos. Se llevó a cabo un experimento en invernadero y otro en condiciones de campo, evaluando el comportamiento de ácidos hidroxicarboxílicos, como biorreguladores orgánicos en plantas de tomate tratadas con aplicación foliar de Metsulfuron-metil y Metribuzina a dosis comercial y sobredosis. En la etapa de invernadero, se evaluó la eficiencia cuántica, contenido de clorofilas, conductancia estomática e intercambio de $\mathrm{CO}_{2}$; en campo, se caracterizó el desarrollo foliar, crecimiento de raíces, tasa de asimilación neta (TAN), tasa relativa de crecimiento (TRC) y rendimiento del cultivo bajo un sistema de producción convencional. La aplicación de Metsulfuron-metil (comercial y sobredosis) no alteró significativamente el contenido de clorofilas ni la conductancia estomática; sin embargo, disminuyó en $80 \%$ y $100 \%$ la eficiencia cuántica e intercambio de $\mathrm{CO}_{2}$ respectivamente, lo cual fue mitigado por la aplicación del biorregulador. Los ácidos hidroxicarboxílicos también evitaron la disminución del crecimiento de raíces y recuperaron la TAN y TRC durante el primer mes de evaluación, propiciando mayor llenado de fruto. Metribuzina afectó significativamente el intercambio de $\mathrm{CO}_{2}$ durante los primeros 15 días después de aplicación de los tratamientos (dda); la aplicación en sobredosis afectó la eficiencia cuántica a partir de 7 dda y causó defoliación a partir de 30 dda, lo que estuvo ligado con un descenso del $20 \%$ en producción. Los biorreguladores disminuyeron el estrés causado por sobredosis de Metribuzina y con el uso se alcanzaron valores similares a las plantas testigo en todas las variables analizadas.

\footnotetext{
Comercial Agrooriente S.A.S., Bogotá (Colombia).

Facultad de Ciencias Agrarias, Departamento de Agronomía, Universidad Nacional de Colombia, Bogotá (Colombia). Autor para correspondencia.gaplazat@unal.edu.co
} 


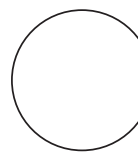

Palabras clave adicionales: Metsulfuron-Metil, Metribuzina, flujo de electrones, ALS, ciclo de Krebs, ciclo de Calvin.

\section{ABSTRACT}

Losing herbicide selectivity is a permanent risk in high-value crops, which is why it is important to have alternatives that mitigate the effect on the physiology of stressed crops. A greenhouse and a field experiment were performed to evaluate the behavior of hidroxy-carboxylic acids (HCA) as an organic bio-regulator in tomato plants that were treated with Metsulfuron-Metil and Metribuzine at regular and excessive doses. In the greenhouse experiment, the quantum efficiency (Fv/Fm), chlorophyll content, stomatal conductance and $\mathrm{CO}_{2}$ exchange were evaluated; in the field experiment, we evaluated the foliar area, root growth, net assimilation rate (NAR), relative growth rate (RGR) and yield. Statistically, the Metsulfuron-metil (regular and excessive rates) did not affect the chlorophyll content, but it decreased the quantum efficiency and $\mathrm{CO}_{2}$ exchange in $80 \%$ and $100 \%$ of the plants, which was alleviated by the bio-regulator application. During the first month after the application, the hidroxy-carboxylic acids did not have the decreased root growth that was seen after the Metsulfuron application, and aided in the recovery of the NAR and RGR, resulting in a better fruit set. The Metribuzine affected the $\mathrm{CO}_{2}$ exchange significantly during 15 days after the application (daa) of treatments whereas the excessive dose application impacted the quantum efficiency for 7 daa and was responsible for leaf fall at 30 daa and 20\% less production. All of the excessive Metribuzin dose injuries were mitigated by the bio-regulator application, reaching similar values as the control plants.

Additional keywords: Metsulfuron-Metil, Metribuzine, electron flux, ALS, Krebs cycle, Calvin cycle.

Fecha de recepción: 18-12-2015

Aprobado para publicación: 14-05-2016
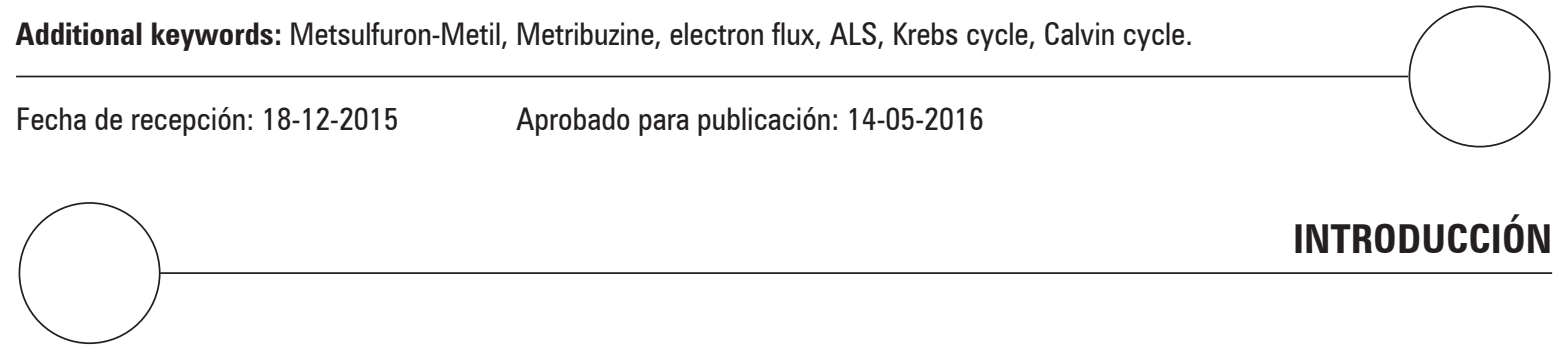

INTRODUCCIÓN

La FAO clasifica al tomate (Solanum lycopersicum L.) como la hortaliza más importante en el mundo, ocupando cerca de 4 millones de hectáreas al igual que estima para 2013 una producción anual de 683.538 t en aproximadamente 16.700 ha, convirtiendo a esta hortaliza en el producto más importante en volumen (Faostat, 2013).

La fitoprotección del cultivo incluye el manejo de artrópodos, hongos y plantas no deseadas. La presencia de malezas durante los primeros 45 días (d) después de trasplante afecta la calidad y el rendimiento hasta en un $90 \%$ (Adigun, 2005) por lo cual es necesario un plan de manejo integrado.

Las solanáceas, incluyendo el tomate, son plantas susceptibles a los herbicidas, por lo cual las posibilidades de daño al cultivo son permanentes, debido tanto a la baja selectividad como al manejo y la aplicación del producto (Ormeño et al., 2002).

En Colombia, el manejo de malezas en el sistema de producción de tomate comprende el uso de prácticas culturales como desyerbas, aporques y aplicación de Metribuzina como la única molécula selectiva registrada en el país ante el ICA para el control de especies latifoliadas en postrasplante del cultivo (ICA, 2016)

De otro lado, la investigación para el uso de otros herbicidas ha sido extensa en países de mayor producción de tomate. En Chile, buscando alternativas para la rotación de Metribuzina, se 
ha evaluado el uso de halosulfuron-metil, el cual causa daños temporales en follaje, con posterior recuperación a los 21 días después de aplicación (Ormeño et al., 2002). Jennings (2010) evaluó sulfonilureas en Carolina del Norte, encontrando que imazasulfuron, halosulfuron y trifloxisulfuron no presentaban efectos negativos sobre la producción, a diferencia de lo comentado por Dittmar et al. (2012) quienes evaluando los mismos herbicidas en postrasplante reportan daños en las plantas de cultivo entre $20 \%$ y $50 \%$ afectando el crecimiento, tras la aplicación en postrasplante de estas moléculas.

Halosulfuron, imazasuluron y trifloxiulfuron son herbicidas del grupo de las sulfonilureas que inhiben la ALS (acetato-lactato sintetasa), la cual es la primera enzima que cataliza la biosíntesis de los aminoácidos valina, leucina e isoleucina, esenciales en el desarrollo vegetal y cuya biosíntesis ocurre principalmente en meristemos, razón por la cual, inicialmente se observan síntomas de daño en tejidos nuevos como la disminución en crecimiento y clorosis (Zhou et al., 2007).

Metribuzina, por su parte, es un herbicida del grupo de las triazinas que afecta la actividad del fotosistema II (PSII), inhibiendo la proteína D1, lo que causa disminución del flujo de electrones y daño oxidativo en el fotosistema, generando estrés de tipo fotoquímico (Idedan et al., 2011).

Este daño puede ser causado en plantas de tomate, bien sea porque se pierde la selectividad por mecanismo de acción en el caso de Sulfonilureas o por sobredosis en el caso de Metribuzina, y para contrarrestarlo existe la alternativa de utilizar ácidos orgánicos, los cuales han demostrado mitigar el efecto negativo del estrés causado por condiciones adversas sobre la producción (ElGhamry et al., 2009).

Slapakauskas et al. (2006) comentaron que los ácidos carboxílicos son una alternativa utilizada para mejorar las características fisiológicas de plantas, optimizando la fotosíntesis e incre- mentando la formación de raíces adventicias, propiciando plantas menos susceptibles y con mayores rendimientos. De igual manera, se reporta que la aplicación de estos ácidos ha aumentado la concentración de clorofilas, logrando incrementar la fotosíntesis en plantas de trigo estresadas (Bahari et al., 2013).

Recientemente se ha investigado el uso de ácidos hidroxicarboxílicos de origen bacteriano. Meike (2008) describe el proceso de producción mediante fermentación bacteriana como potencial activador del metabolismo vegetal a través de diferentes vías: como reguladores del $\mathrm{pH}$ celular, lo que protege la actividad enzimática (Mackowiak et al., 2001); como inductores de la síntesis de polioles que hidratan las células y actúan como precursores de azúcares neutros (Kudrev y Petrova, 1975); como reguladores de los canales de $\mathrm{Ca}^{2+}$ y trasporte de ADN en la célula (Dawes y Senior, 1973) y como precursores de la translocación de asimilados, propiciando mejor formación de raíces y mayores rendimientos (Slapakauskas et al., 2006; Higa y Kinjo, 1991).

En este trabajo se evaluó el efecto de ácidos hidroxicarboxílicos, producidos a partir de la fermentación de cascarillas agrícolas, como biorreguladores del estrés causado por Metsulfuron-metil (Sulfonilurea) y Metribuzina (Triazina) aplicados en postrasplante del cultivo de tomate.

\section{MATERIALES Y MÉTODOS}

\section{Intercambio gaseoso y fluorescencia de clorofilas en condiciones de invernadero}

Este ensayo se llevó a cabo en el invernadero de Malherbología en la Universidad Nacional de Colombia, sede Bogotá (4³8'10.96"N7405'14.92"O), durante 45 d. De estos, los primeros $17 \mathrm{~d}$ corresponden al establecimiento, y los siguientes 28, al tiempo de evaluación. La temperatura y humedad relativa (HR) fueron controlados en el invernadero mediante sistemas 
de ventilación y calefacción (temperatura: máx. 32 y mín. $22^{\circ} \mathrm{C}$; HR $85 \%$ ).

Se evaluaron plantas de tomate híbrido Calima tipo santa Clara, las cuales fueron trasplantadas con cuatro hojas verdaderas, en materas de $2 \mathrm{~kg}$ de capacidad, en suelos FA pasteurizado. Posteriormente, las materas se ubicaron según un diseño completamente al azar (DCA) con nueve tratamientos y cuatro repeticiones, llevándose a cabo cinco muestreos destructivos (24 horas (h); 7, 15, 21 y 28 d después de aplicación de los tratamientos, dda).

Los tratamientos del uno al cuatro consistieron en dos herbicidas (metribuzina y metsulfuron) a dosis comercial ( $\mathrm{X}=336 \mathrm{~g} \mathrm{ha}^{-1}$ y $9 \mathrm{~g} \mathrm{ha}^{-1}$ del i.a., respectivamente) y sobredosis $(2 \mathrm{X}=672$ $\mathrm{g} \mathrm{ha}^{-1}$ y $18 \mathrm{~g} \mathrm{ha}^{-1}$ del i.a., respectivamente). Los tratamientos del cinco al ocho correspondieron a los tratamientos anteriores más la adición de ácidos hidroxicarboxílicos AHC (a dosis comercial, $2 \mathrm{~L}^{\text {ha }}{ }^{-1}$ ATPup $\left.®\right)$ y finalmente un tratamiento nueve, considerado control (sin adición alguna).

A los 20 d después de trasplante, se realizó la aplicación de herbicidas mediante el uso de pulverizadora automática con boquilla $0,5 \mathrm{~T}$-jeet de abanico plano. Posteriormente y con el ánimo de evitar alteración química de los herbicidas con los biorreguladores, 2 h después de la aplicación de los mencionados primero, se realizó la aplicación de AHC con boquilla 0,4 T-jeet de cono lleno.

Las mediciones de intercambio de $\mathrm{CO}_{2}(\mu \mathrm{mol}$ $\left.\mathrm{m}^{-2} \mathrm{~s}^{1}\right)$ y conductancia estomática $\left(\mathrm{cm} \mathrm{s}^{1}\right)$ se realizaron con el equipo LI-COR 6.200 (Licor, Lincoln, NE); y eficiencia cuántica máxima (Fv/ Fm) con el fluorómetro Handy Pea (Hansatech Instruments, Norfolk, UK). Estas variables se evaluaron a las 24 h; 7, 15, 21 y 28 dda entre las 9:00 y 10:00 a.m.; tomando como muestra la cuarta completamente desarrollada. El contenido de clorofilas ( $\mathrm{mg} \mathrm{g}^{-1}$ de peso fresco) se midió con espectrofotómetro de absorción BioMateTM
(Thermo Electron Corporation, Waltham, MA) a los 7, 15, 21 y 28 dda mediante el método de espectrofotometría descrito por MacKinney (1941).

Cada variable fue analizada en el software estadístico R. 3.01 como un DCA y posteriormente se realizó la prueba de comparación de medias de Tukey para obtener el resultado de diferencias significativas de los datos $(P \leq 0,05)$.

\section{Evaluación de crecimiento y producción en campo}

El segundo ensayo se desarrolló en el municipio de Cáqueza-Cundinamarca, vereda Pantano de Carlos, finca E1 Riego $\left(4^{\circ} 24^{\prime} 1,21^{\prime \prime} \mathrm{N}\right.$ y 7357'4,78"W) a $1.750 \mathrm{msnm}$, bajo condición de bosque seco premontano (bs-PM) en suelo FA, predominante inceptisol -según la unidad cartográfica: Estudio de suelos y zonificación de tierras de Cundinamarca IGAC (2000), durante 4 meses, tiempo para obtener la primera cosecha completa del primer racimo productivo.

El ensayo se elaboró a campo abierto, bajo las mismas condiciones de un cultivo comercial de la región (30.000 plantas/ha), utilizando plántulas con cuatro hojas verdaderas de tomate híbrido Calima tipo Santa Clara.

Se realizó un diseño en parcelas divididas con bloques completos al azar en tres repeticiones. Las parcelas principales con una población de 100 plantas correspondieron a la aplicación de los dos herbicidas a dosis comercial (la cual se definirá en este artículo como $\mathrm{X}$ ), sobre dosis (la cual será denotada como $2 \mathrm{X}$ y corresponde al doble de la dosis comercial) y un control (sin aplicación de herbicidas); las subparcelas por su parte representaron la aplicación o no de AHC (2 y $0 \mathrm{~L} \mathrm{ha}^{-1}$ ). Por su parte el bloque se realizó teniendo en cuenta la irregularidad topográfica del lote, dividiendo en tres bloques.

Los herbicidas se aplicaron sobre plantas con 30 días de trasplante, realizando la actividad de ma- 
nera localizada a la base de las plantas de tomate, con boquilla de abanico plano 0,5 T-jeet.

Los AHC fueron aplicados en dos momentos y de manera foliar sobre las plantas de tomate: 25 días de trasplante $(5 \mathrm{~d}$ antes de la aplicación de herbicidas) y $35 \mathrm{ddt}$ ( $5 \mathrm{~d}$ después de la aplicación de herbicidas). La dosis utilizada fue $2 \mathrm{~L} \mathrm{ha}^{-1}$ según recomendación del producto y la actividad se desarrolló con boquilla de cono lleno 0,4 T-jeet.

El primer muestreo se realizó el día de aplicación de herbicidas, y posteriormente cuatro muestreos con intervalo $15 \mathrm{~d}$ hasta alcanzar los 60 dda, momento en que ocurrió la madurez del primer racimo productivo. Las medidas fueron: área foliar, peso seco de raíz y peso seco de parte aérea, variables con las que se calcularon índices de crecimiento como tasa relativa de crecimiento (TRC) y tasa de asimilación neta (TAN). A los 60 dda se realizó cosecha del primer racimo, evaluando el peso fresco del mismo.

El análisis estadístico para cada una de las variables se realizó en el software estadístico $\mathrm{R}$, con prueba de comparación entre parcelas principales, prueba de Tukey y análisis del efecto de la subparcela $(P \leq 0,05)$ según un modelo de regre-

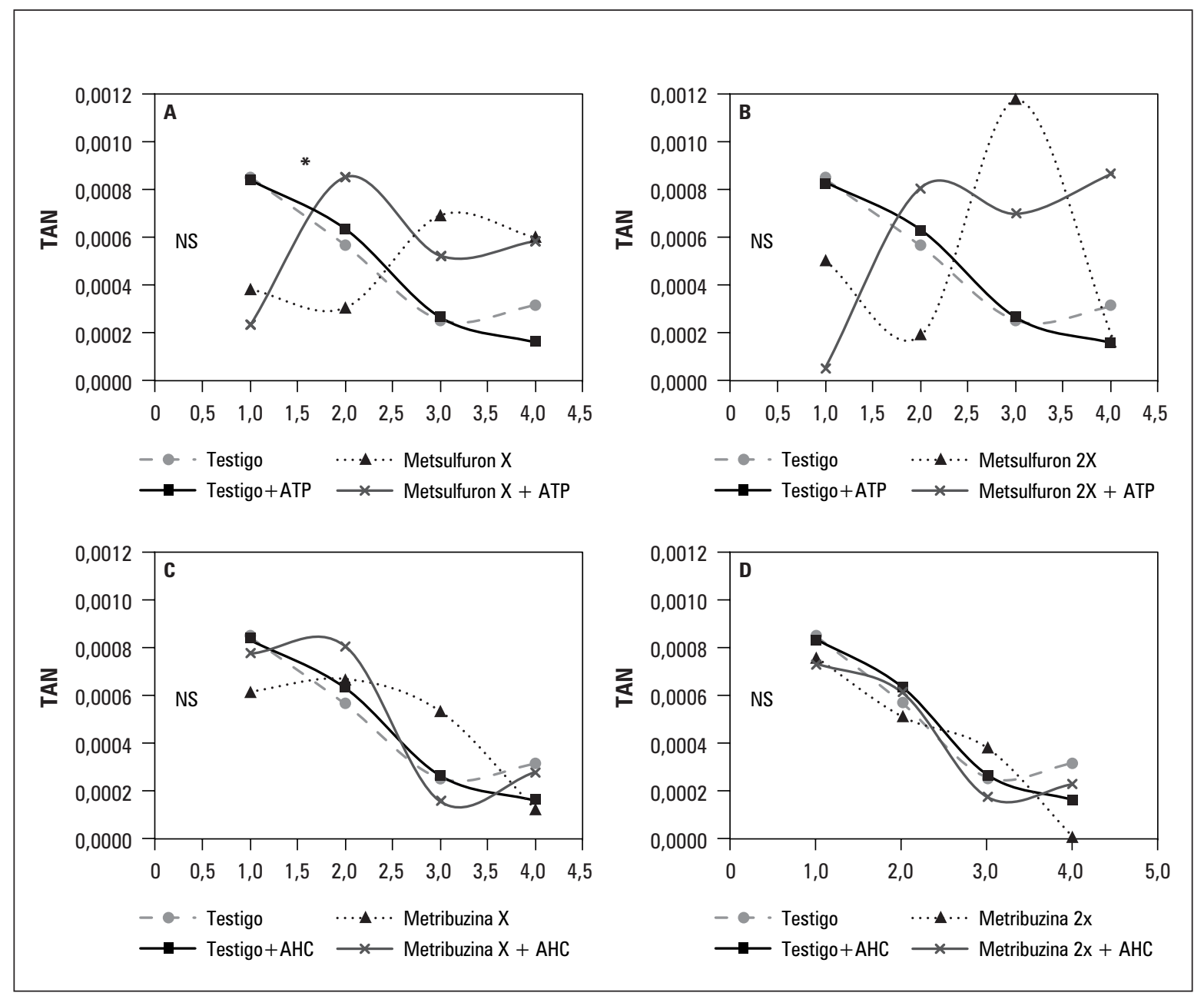

Figura 1. Tasa de asimilación neta (TAN, $\mathrm{g} \mathrm{cm}^{-2}$ día $\left.^{-1}\right)$ en plantas de tomate, en cuatro épocas $(1=0-15 \mathrm{~d}, 2=$ 15-30 d, $3=30-45 \mathrm{~d}$ y $4=45-60 \mathrm{~d}$ ), tratadas con A) Metsulfuron a dosis comercial, B) Metsulfuron en sobredosis, C) Metribuzina en dosis comercial, D) Metribuzina en sobredosis. Todas las subfiguras están relacionadas con el testigo, con y sin aplicación de AHC. NS representa la no significancia entre parcelas $y *$ indica efecto significativo de la subparcela $(P \leq 0,05)$. 
sión lineal con prueba $T$, el cual permite analizar con una ecuación de primer grado, la correlación entre dos variables y/o parcelas (Pardoe, 2012).

\section{RESULTADOS Y DISCUSIÓN}

\section{Intercambio gaseoso y fluorescencia de cloro- filas en condiciones de invernadero}

De acuerdo a los resultados obtenidos, el mayor nivel de estrés lo presentaron las plantas tratadas con Metsulfuron (en dosis comercial y sobredosis) presentando valores $\mathrm{Fv} / \mathrm{Fm}$ de 0,2 a los 21 y 28 dda (tabla 1). Esto podría estar relacionado con el efecto de Metsulfuron en la inhibición de ALS, lo que según Preston et al. (2006) al presentarse mutaciones en la biosíntesis de serina y prolina, componentes de la proteína $\mathrm{D} 1$, se ve afectado el flujo de electrones del PSII. Menegat et al. (2012) reportan disminución en la eficiencia cuántica $(<0,2)$ sobre especies sensibles a sulfonilureas, lo cual podría relacionar al tomate híbrido Calima como un cultivar sensible a este grupo de herbicidas.

La aplicación de AHC sobre las plantas tratadas con Metsulfuron (dosis comercial y sobredosis) presenta valores de $\mathrm{Fv} / \mathrm{Fm}$ significativamente mayores a los $21 \mathrm{dda}$, aunque inferior al valor ideal (tabla 1). Lo anterior permite suponer disminución de los daños causados por el herbicida en el PSII. Esta recuperación estaría relacionada con un aumento en la actividad del ciclo de Calvin que genera la aplicación de los AHC, lo cual aumenta la eficiencia de la energía en el metabolismo, y por ende recuperación más rápida ante condiciones de estrés (Slapakauskas et al., 2006).

Los valores encontrados en el tratamiento de Metribuzina a dosis comercial no afectó la eficiencia cuántica, lo que corrobora la selectividad del herbicida al cultivo. Sin embargo, este mismo herbicida en sobredosis disminuye los valores de Fv/Fm entre 0,2 y 0,6 (tabla 2). Lo anterior, producto de la afectación en el flujo de electrones en el PSII a nivel de la proteína D1 y la disminución

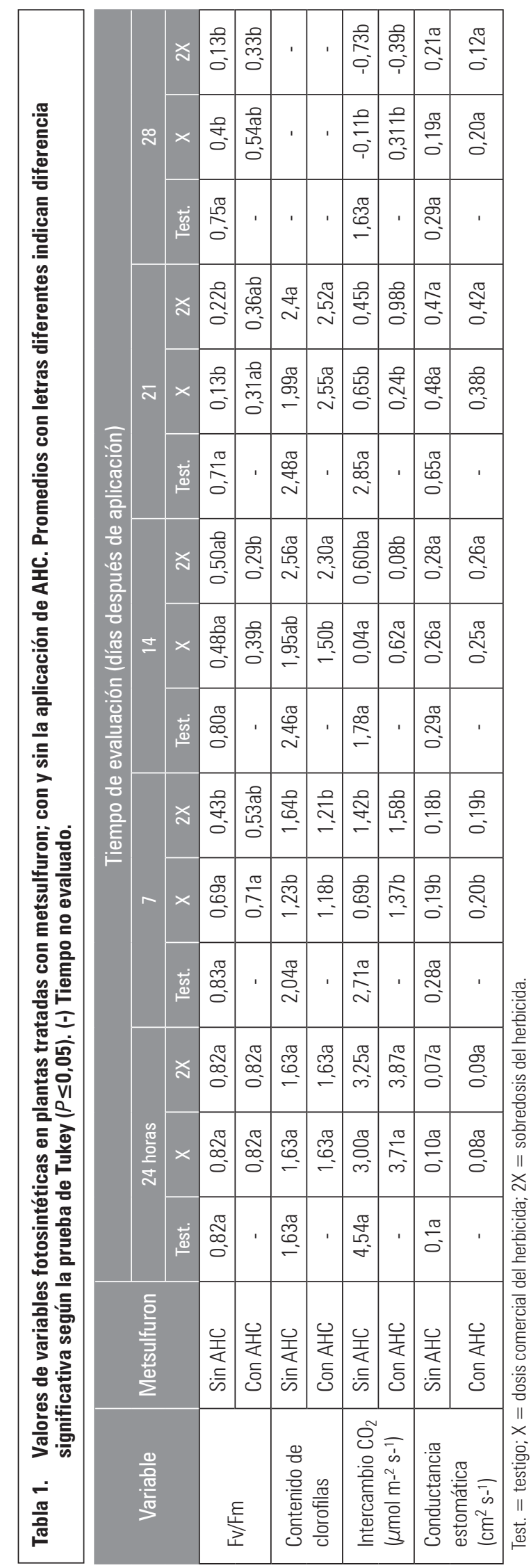


en la eficiencia de la captación de luz (Idedan et al., 2011). Aunque la planta tiende a recuperarse gradualmente a partir de $21 \mathrm{dda}$, los valores se mantuvieron por debajo de 0,8 . Contrarrestando el daño causado por la sobredosis de la triazina a nivel del PSII, la aplicación de AHC logró mantener $\mathrm{Fv} / \mathrm{Fm}$ cercana a 0,8 con valores incluso mayores a los obtenidos por las plantas testigo (tabla 2); lo anterior significa que la detención del flujo de electrones que generan los herbicidas inhibidores de $\mathrm{D} 1$ puede ser mitigada completamente con el uso de AHC en plantas de tomate.

La afectación del Metsulfuron es apreciable a partir de los 7 ddt (disminución con respecto al valor de referencia de 0,89 ) a diferencia de la Metribuzina, en el cual solo se presenta una leve disminución de 0,26 en esta misma evaluación. Con el uso de los AHC esa tendencia se incrementa en Metsulfuron pero decrece para Metribuzina, inclusive en sobredosis, presentando la mayor disminución con valor de 0,6 a los 21 ddt (tablas 1 y 2).

Los resultados en el contenido de clorofila muestran que a pesar de existir diferencias en cuanto el modo y mecanismo de acción entre Metribuzina y Metsulfuron, los dos herbicidas presentan tendencias similares en la disminución del contenido de clorofilas para las dos dosis, causando disminución significativa desde 15 hasta 28 dda. Lo anterior indica que a través de diferentes rutas, Metribuzina y Metsulfuron afectan el contenido de clorofila en magnitudes similares (tablas 1 y 2).

Para el caso de Metsulfuron, Keutgen et al. (1999) descubrieron que la aplicación de este herbicida disminuye la asimilación y contenido de nitrato en plantas. Esto podría explicar una disminución en la formación de clorofilas, cuya molécula contiene $\mathrm{N}$ como elemento estructural (Taiz y Zeiger, 2010). De otro lado, la disminución en el contenido de clorofilas por parte de Metribuzina podría estar relacionada con especies reactivas de oxígeno (ROS), que se forman al interrumpirse el flujo de electrones en el PSII, generando peroxida-

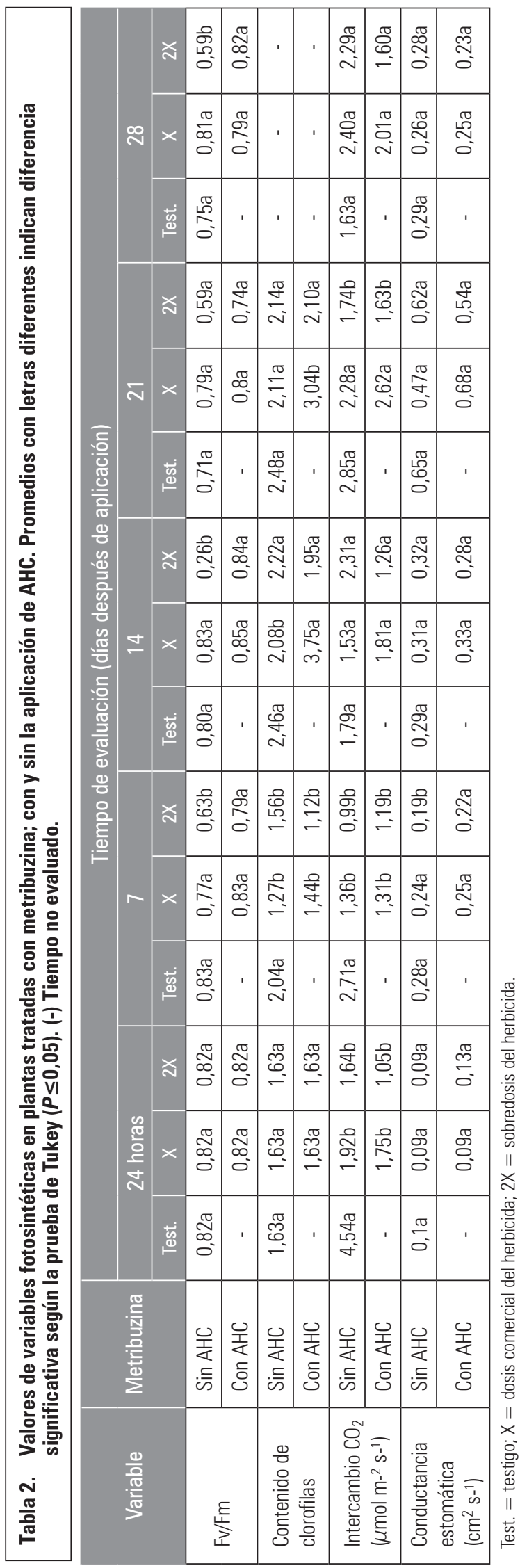


ción de lípidos y daños en membranas, incluidos los tilacoides, ocasionando degradación de clorofilas (Mittler, 2002; Nemat y Hassan, 2006).

Los resultados muestran el efecto positivo de los AHC en la dosis comercial para los dos herbicidas. En el caso de Metsulfuron, la aplicación del biorregulador logró restablecer el contenido de clorofilas con respecto a las plantas testigo entre 21 y 28 dda. Para las plantas tratadas con Metribuzina en dosis comercial, el contenido de clorofilas aumentó hasta un $75 \%$ tras la aplicación de AHC y $50 \%$ con respecto a las plantas testigo (tabla 2). Lo anterior corrobora la ventaja en el uso de ácidos orgánicos para la formación de clorofilas, propiciado por el aumento en la actividad enzimática y mayor absorción de nutrientes como nitrógeno (Slim et al., 2012). Sin embargo, la sobredosis de Metribuzina causó mayor daño en plantas, limitando la acción benéfica de los AHC en la formación de clorofilas, generando resultados sin diferencias significativas comparados con Metribuzina 2X.

Con relación al intercambio de $\mathrm{CO}_{2}$, los valores en plantas testigo oscilaron entre 1,79 y 4,54 $\mu \mathrm{mol} \mathrm{m} \mathrm{s}^{-1}$; sin embargo, la aplicación de herbicidas ocasionó un comportamiento diferente en la fotosíntesis de las plantas. Tras la aplicación de Metribuzina y Metsulfuron los valores de tasa fotosintética disminuyeron significativamente a los 7 dda. A pesar de que Metribuzina afectó con mayor rapidez la fotosíntesis (1 dda), las plantas lograron recuperarse a partir de 15 dda hasta alcanzar valores cercanos a las plantas testigo (tabla 2). Esto demuestra la capacidad que tienen las solanáceas de contrarrestar el daño causado por triazinas mediante un proceso conocido como metabolización de la molécula (Gang et al., 2011), lo cual consiste en la formación de conjugados de glucosa-Metribuzina y N-glucósidos-Metribuzina, limitando el efecto del herbicida dentro de la planta (Smith et al., 1989).

La aplicación de Metsulfuron disminuyó significativamente la absorción de $\mathrm{CO}_{2}$ a partir de 7 dda y continuó afectando hasta alcanzar valores negativos a los 28 dda. Riethmuller et al. (2006)

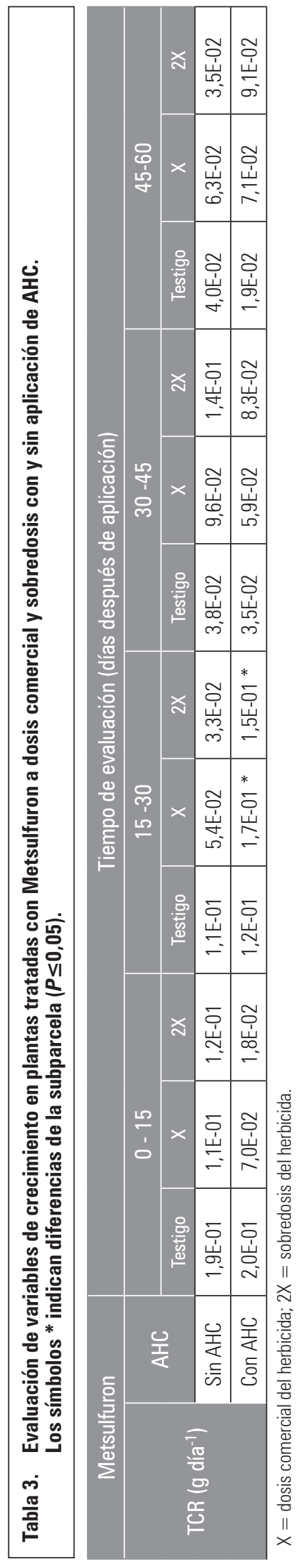

Vol. 10 - No. 1 - 2016 
encontraron que en tomate la aplicación de Metsulfuron-Metil causa disminución de intercambio de $\mathrm{CO}_{2}$ hasta en un $60 \%$, relacionando este efecto con el aumento de la clorosis intervenal que se genera en el proceso de marchitez foliar, cuando el nivel de estrés es tal que causa muerte gradual y se disminuye la fotosíntesis aumentando la respiración en el tejido afectado. Esto podría explicar el hecho de que en este ensayo se alcanzaran valores críticos $<0$ para las plantas tratadas con Metsulfuron (muerte de tejido foliar) durante el primer mes después de aplicación (tabla 1).

No se presentó efecto significativo de los AHC $(P>0,05)$ en la fotosíntesis de las plantas estresadas; sin embargo, en las tablas 1 y 2 se aprecia la tendencia a incrementar la fijación de $\mathrm{CO}_{2}$ para los tratamientos Metribuzina X + AHC y Metsulfuron X + AHC. A pesar de que la ficha técnica del producto biorregulador ofrece la posibilidad de restablecer la actividad metabólica en plantas estresadas (Innovak Global, 2013), en el presente ensayo no se presentaron diferencias significativas en la regulación del estrés que generó la aplicación de metribuzina y metsulfuron en su dosis comercial con respecto a esta variable.

En cuanto a los valores de conductancia estomática el efecto de los tratamientos encontró que Metsulfuron generó mayor nivel de estrés con diferencias significativas a los 7 dda para la dosis comercial y sobredosis (tabla 1). Otras sulfonilureas como Imazosulfuron y trifloxysulfuron han presentado la misma tendencia, disminuyendo la conductancia estomática de plantas de tomate desde 7 hasta 16 dda (Dittmar et al., 2012), aunque específicamente en Metsulfuron el cierre estomático no ha sido muy estudiado. Ashcroft y Ashcroft (1990) sugieren que las sulfonilureas son un potente bloqueador de los canales K-ATP en numerosos tejidos, lo cual afecta directamente el movimiento de las células guarda en plantas, y podría ser la causa del cierre estomático en este ensayo.

La aplicación de Metribuzina a la dosis recomendada no afectó el comportamiento de esta varia-

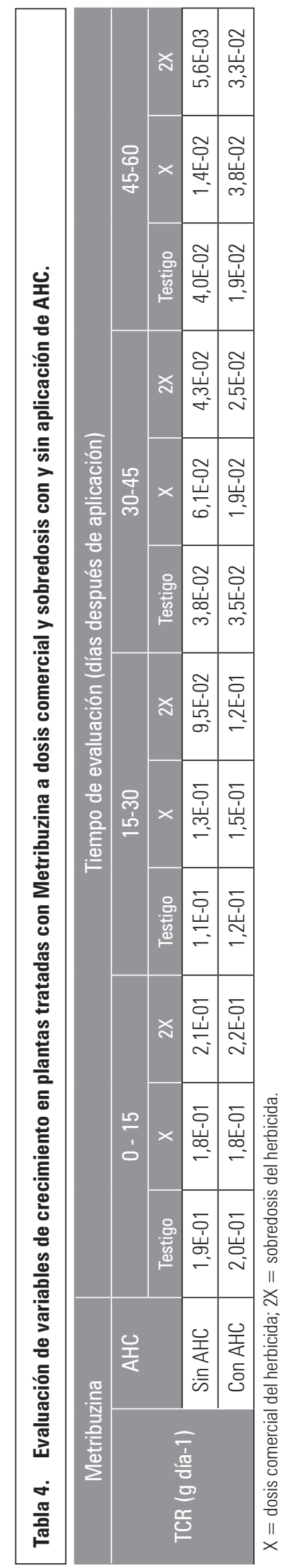


ble, lo que nuevamente destaca la selectividad del herbicida a esta especie de cultivo. No obstante, la dosis $2 \mathrm{X}$ causó disminución de la conductancia estomática a 7 dda (tabla 2), lo cual concuerda con el inicio de daño en el PSII, según lo observado en eficiencia cuántica Fv/Fm. Esta relación demuestra que el daño en el fotosistema II es un indicativo del estrés, donde el daño a nivel energético puede generar un desbalance en la cantidad de carbono interno y disminuir la conductancia de estomas (Else et al., 2009).

Los AHC no alteraron significativamente los resultados de conductancia estomática en plantas tratadas con herbicidas, indicando que estos biorreguladores fueron menos eficientes en la protección de los canales de $\mathrm{K}^{+}$(para el caso de Metsulfuron) o que el estrés que generaron los herbicidas pudieron inducir la síntesis de ABA. Teniendo en cuenta que la biosíntesis de esta hormona promueve el cierre estomático (Taiz y Zeiger, 2010) y los resultados encontrados no muestran diferencia de la apertura estomática e intercambio de $\mathrm{CO}_{2}$, se sugiere ahondar en el estudio del efecto fisiológico de los AHC.

\section{Evaluación del crecimiento y producción en campo}

El comportamiento general de la TAN muestra que la eficiencia fotosintética disminuye a lo largo del tiempo (figura 1), como un comportamiento natural que se explica con el cambio en la vocación de las células, disminuyendo el crecimiento y aumentando la diferenciación para la formación de flores y frutos. La misma dinámica de TAN ha sido encontrada por Barraza et al. (2004) en Colombia.

No se observaron diferencias significativas en la TAN ni efecto de los AHC para las plantas tratadas con Metsulfuron 2X (figura 1B). Sin embargo, la figura 1A muestra que la tendencia es similar a lo presentado por Metsulfuron X, en el cual se disminuyó la eficiencia fotosintética en $50 \%$ para el primer mes de evaluación y ocu-

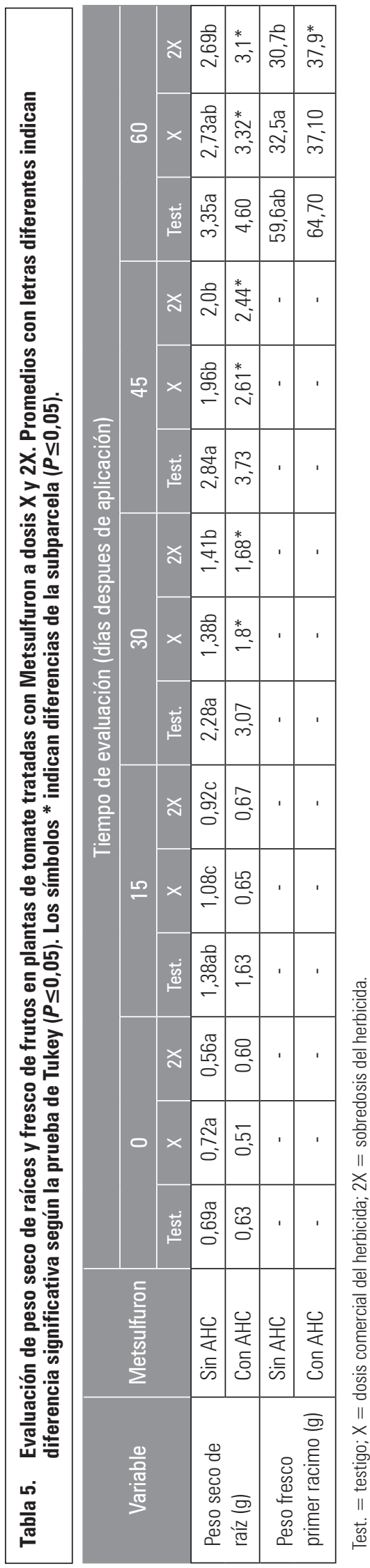

Vol. 10 - No. 1 - 2016 
rrió recuperación durante el segundo (figura 1A), brindando información de que las plantas de tomate para el caso de este ensayo toman $30 \mathrm{~d}$ en iniciar una recuperación de forma natural tras el estrés causado por la sulfonilurea.

Por su parte, la dosis comercial de Metribuzina no afectó significativamente el comportamiento de la TAN. Se aprecia una ligera disminución de la eficiencia fotosintética en los primeros $15 \mathrm{~d}$, aunque la tendencia se normalizó 30 dda (figura 1C). Para el caso de Metribuzina en sobredosis, la figura 1D muestra la disminución de la TAN entre 7 y 15 dda, lo cual coincide con el estrés presentado por las plantas en la eficiencia cuántica (Fv/Fm) en este mismo periodo; confirmando la relación que existe entre la integridad del PSII con la eficiencia fotosintética (Taiz y Zeiger, 2010). La aplicación de AHC logró mantener una curva con comportamiento normal sobre las plantas tratadas con Metribuzina, tal como logró mantener la integridad del PSII (tablas 2 y 4).

La comparación entre testigo con o sin AHC y la aplicación de Metsulfuron con o sin AHC presenta mayor tendencia a cambio, que la comparación entre testigo con o sin AHC y la aplicación de Metribuzina con o sin AHC. Esta tendencia podría explicarse por las diferencias en los niveles de afectación "estrés" de cada uno de los herbicidas.

Por otra parte, la tasa relativa de crecimiento (TRC) comparte la misma tendencia a lo observado en la TAN. En general las plantas testigo disminuyeron la TRC durante el tiempo de evaluación. Barranza et al. (2004) relacionaron la TAN con el proceso de producción de asimilados, responsable del crecimiento en plantas de tomate; en ese sentido, cuando la TAN disminuye, también decrece la TRC.

La aplicación de Metsulfuron en dosis comercial y sobredosis no generaron diferencias significativas en la TRC; sin embargo, se observó disminución del crecimiento en los primeros 30 dda para las dos dosis. Los AHC aumentaron la TRC de

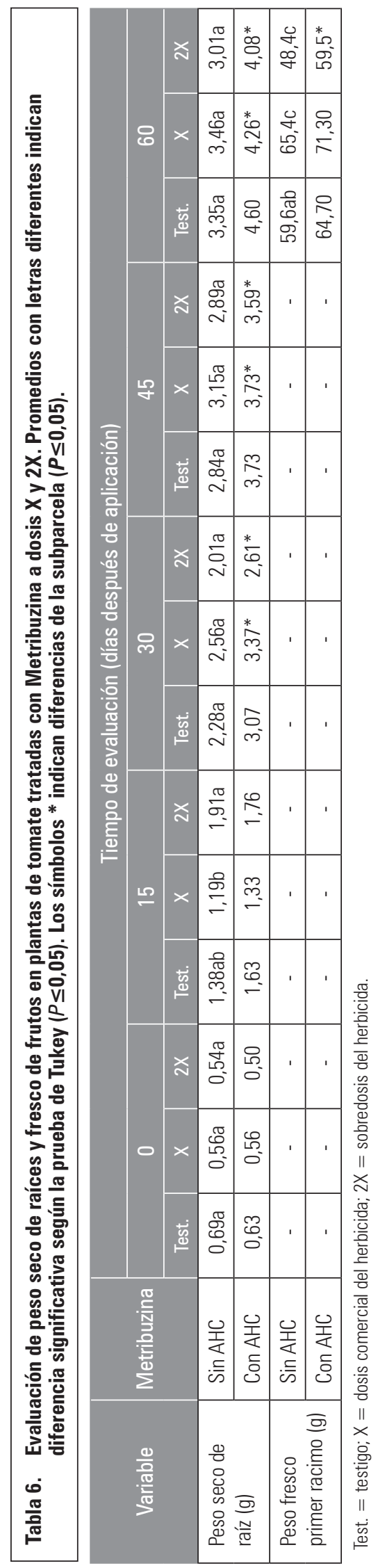


las plantas tratadas con Metsulfuron $(P \leq 0,05)$ entre 15 y $30 \mathrm{dda}$. A partir de $30 \mathrm{dda}$ el comportamiento de las plantas tratadas con Metsulfuron + AHC comparten la misma tendencia con las plantas testigo, ratificando que los biorreguladores pueden propiciar reactivación metabólica tal como se observó en el crecimiento de raíces (Meike, 2008).

Los AHC no presentaron diferencias significativas en la dinámica de crecimiento en plantas tratadas con Metribuzina y plantas testigo (tabla 4). Esto indica que las ganancias obtenidas en el peso seco de raíz (tabla 6) corresponden más a una dinámica de translocación que a una ganancia de peso general de la planta, por lo cual tampoco se presentaron diferencias en la TAN y TRC para estos tratamientos. Esta dinámica también fue encontrada por Slapakauskas et al. (2006), donde los ácidos carboxílicos mejoraron tanto la eficiencia fotosintética como la formación de raíces en plantas de frijol.

El rendimiento del tomate se vio afectado drásticamente por la aplicación de Metsulfuron, causando disminución de 46 y 49\%, para la dosis comercial y sobredosis respectivamente (tabla 5). El llenado de fruto pudo verse afectado por el déficit de translocación que causan los inhibidores de ALS en plantas susceptibles. Bestman et al. (1990) encontraron que la aplicación de sulfonilureas ocasionaba acumulación de compuestos orgánicos en las hojas de Thiasp sp., disminuyendo la tasa de translocación de azúcares, lo que afecta el rendimiento de cultivos (Zhou et al., 2007). Para el caso de este trabajo no se evaluó peso seco de hojas por separado, por lo cual no es posible relacionar con veracidad el proceso de translocación con el llenado de fruto; sin embargo, los bajos valores obtenidos en la TAN a los 20 dda (figura 1) indican el bajo estrés el cual se concentraba en las plantas en el tiempo inicial del llenado de fruto, lo cual pudo afectar el rendimiento.

Por otro lado, la aplicación de AHC aumentó el rendimiento para todos los tratamientos. El daño causado por Metsulfuron fue disminuido tras la aplicación del biorregulador en 14 y 23\% para la dosis comercial y sobredosis respectivamente, con diferencias significativas en el último caso. Así mismo, aumentó el rendimiento de las plantas tratadas con sobredosis de Metribuzina, logrando valores similares a las plantas testigo

El efecto positivo de los biorreguladores puede relacionarse al proceso de hidratación celular, lo cual favorece la división y el crecimiento de las mismas (Innovak Global, 2013), y/o a la protección que generan sobre la maquinaria enzimática y protéica (Meike, 2008), algunas de las cuales están directamente relacionadas con el desarrollo de frutos, tal como afirmaron Steinhauser et al. (2010), quienes encontraron más de 22 enzimas relacionadas con la fotosíntesis, glicolisis, translocación, entre otros, expresadas durante el llenado de fruto.

\section{CONCLUSIONES}

El herbicida Metribuzina en dosis comercial fue selectivo al cultivo de tomate; las variables relacionadas con fotosíntesis indicaron que la planta es capaz de mitigar el estrés que causa la molécula, evitando el daño en el PSII y propiciando un crecimiento normal de la planta.

La sobredosis de Metribuzina generó daños significativos en el PSII disminuyendo la eficiencia cuántica, intercambio gaseoso y rendimiento en plantas de tomate.

Las plantas de tomate mostraron susceptibilidad a la aplicación de Metsulfuron; las dos dosis evaluadas generaron disminución en la eficiencia cuántica, valores de intercambio gaseoso muy cercanos a cero, y disminución en la tasa de asimilación neta, afectando la translocación de asimilados para el crecimiento de raíces y llenado de fruto.

Los ácidos hidroxicarboxílicos aumentaron el contenido de clorofila y mitigaron el daño a nivel del PSII que causa el estrés generado por los her- 
bicidas Metsulfuron y Metribuzina, con mejores resultados para el segundo.

Los ácidos hidroxicarboxílicos aumentaron el peso de raíces de plantas estresadas y no estresadas a partir de $30 \mathrm{dda}$, aumentando las tasas de crecimiento para el mismo periodo.

\section{AGRADECIMIENTOS}

A Innovak Global, México-Colombia, la Ing. Magda Murcia, representante comercial de Innovak Global en Colombia, al Laboratorio de Malherbología, Facultad de Ciencias Agrarias, Universidad Nacional de Colombia, sede Bogotá.

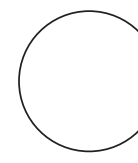

Adigun, J. 2005. Critical period of weed interference in rainfed and irrigated tomatoes in the nigerian savanna. Agricultura tropica et sub-tropica 38(2), 73-80.

Ashcroft, F. y S. Ashcroft. 1990. Properties and functions of ATP-sensitive K-Channel. Cell Signal 2, $197-$ 214. Doi: 10.1016/0898-6568(90)90048-F

Bahari, A., H. Pirdashti y M. Yaghubi. 2013. The effects of amino acid fertilizer spraying on photosynthetic pigments and antioxidant enzymes of wheat (Triticum aestivum L.) under salinity stress. Int. J. Agron. Plant Prod. 4(4), 787-793.

Barranza, F., G. Fischer y C. Cardona. 2004. Estudio del proceso de crecimiento del cultivo del tomate (Lycopersicon esculentum Mill.) en el valle del Sinú medio, Colombia. Agron. Colomb. 22(1), 81-90.

Bestman, H., M. Devine y W. Born. 1990. Herbicide chlorsulfuron decreases assimilate transport out of treated leaves of field pennycress (Thiaspi arvense L.) seedlings. Plant Physiol. 93(1), 1441-1448. Doi: doi.org/10.1104/pp.93.4.1441

Dawes, E.A. y P.J. Senior. 1973. The role and regulation of energy reserve polymers in micro-organisms. Adv. Microb. Physiol. 10, 135-266. Doi: 10.1016/ S0065-2911(08)60088-0

Dittmar, P., D. Monks, K. Jennings y F. Booker. 2012. Tolerance of tomato to herbicides applied through drip irrigation. Weed Technol. 26(4), 684-690. Doi: 10.1614/WT-D-11-00181.1

El-Ghamry, A., K. El-Hai y K. Ghoneem. 2009. Amino and humic acids promote growth, yield and disease resistance of faba bean (Vicia faba L.) cultivated in clayey soil. Aust. J. Basic Appl. Sci. 3(2), 731-739.

Else, M., F. Janowiak, C. Atkinson y M. Jackson. 2009. Root signals and stomatal closure in relation to photosynthesis, chlorophyll a fluorescence and adventitious rooting of flooded tomato plants. Ann. Bot. 103(1), 313-323.
REFERENCIAS BIBLIOGRÁFICAS

FAOSTAT. 2013. Production quantity by crop. FAO statistics division. Food and agricultural organization. En: http://faostat.fao.org; consulta: noviembre de 2015.

Gang, P., S. Ping, Y. Oin, P. Steve y T. Jumin. 2011. Nontarget-site based metribuzin tolerance in two induced mutants of narrow-leafed lupin. En: http:// www. Paper.edu.cn; consulta: octubre de 2013.

Higa, T. y S. Kinjo. 1991. Effect of lactic acid fermentation bacteria on plant growth and soil humus formation. University of the Ryukyus, Okinawa, Japón.

ICA. 2016. Registros nacionales Marzo 2016. Instituto Colombiano Agropecuario. En: http://www. ica.gov.co/getdoc/d3612ebf-a5a6-4702-8d4b$8427 c 1 c d a e b 1 / R E G I S T R O S-N A C I O N A L E S-$ PQUA-15-04-09.aspx; consulta: abril de 2016.

Idedan, I., T. Tomo y T. Noguchi. 2011. Herbicide effect on the photodamage process of photosystem II: Fourier transform infrared study. Biochem. Biophys. Acta 1807(1), 1214-1220.

IGAC. 2000. Estudio general de suelos y zonificación de tierras de Cundinamarca. Instituto Geográfico Agustín Codazzi, Bogotá.

Innovak Global ${ }^{\circ}$. 2013. ATPup, biorregulador fisiológico anti-estresante. En: http://www.innovakglobal.com/productos/atpup; consulta: noviembre de 2013.

Jennings, K. 2010. Tolerance of fresh-market tomato to post-emergence-directed imazosulfuron, halosulfuron and trifloxysulfuron. Weed Technol. 24(1), 117-120. Doi: 10.1614/WT-09-056.1

Keutgen, N., S. Matsuhashi, M. Jia, C. Mizuniwa, T. Ito, T. Fujimura, S. Hashimoto, N. Ishioka, S. Watanabe, A. Osa, T. Sekine y H. Uchida. 1999. Nitrogen metabolism in soybean (Glycine max L.) as affected by metsulfuron-methyl. Acta Hortic. 531(1), 175180. Doi: 10.17660/ActaHortic.2000.531.25 
Kudrev, T. y L. Petrova. 1975. Inflluence of some PGR on water balance. Fiziol. Rast. (Sofia) 4, 71-75.

MacKinney, G. 1941. Absorption oflight by chlorophyll solutions. Biol. Chem. 140(1), 315-322.

Mackowiak, C., P. Grossl y B. Bugbee. 2001. Beneficial effects of humic acid on micronutrient availability to wheat. Soil Science Society of America 65(1),1744-1750. Doi: 10.2136/sssaj2001.1744

Menegat, A., Y. Kaiser, A. Stephan, H. Ni y R. Gerhards. 2012. Chlorophyll fluorescence microscreening as a rapid detection method for herbicide resistance in grass weeds in north china plain winter wheat production systems and beyond. Weed Sci. 18(1), 409-418.

Meike, K. 2008. Production of (R)-3-hydroxycarboxylic acids from bacterial polyhydroxyalkanoates (PHA) and investigation of the physiological role of PHA degradation. Tesis de doctorado. Technical University of Munich, Alemania.

Mittler, R. 2002. Oxidative stress, antioxidants and stress tolerance. Trends Plant Sci. 7(1), 405-410. Doi: 10.1016/S1360-1385(02)02312-9

Nemat, M. y N. Hassan. 2006. Changes of antioxidants levels in two maize lines following atrazine treatments. Plant Physiol. Biochem. 44(1), 202210. Doi: $10.1016 /$ j.plaphy.2006.05.004

Ormeño, J., F. Fuentes y V. Soffia. 2002. Tolerancia del tomate (Lycopersicon esculentum Mill.) a aplicaciones post trasplante del herbicida halosulfuron-metil. Agric. Téc. 63(2), 125-134.

Pardoe. I. 2012. Applied regression modeling. $2^{\text {nd }}$ ed. John Wiley and Sons, New Jersey, NJ. Doi: 10.1002/9781118345054

Preston, C., L. Stone, M. Rieger y J. Baker. 2006. Multiple effects of a naturally occurring proline to threo- nine substitution within acetolactate synthase in two herbicide-resistant populations of Lactucaserriola. Pestic. Biochem. Physiol. 84(1), 227-235. Doi: 10.1016/j.pestbp.2005.07.007

Riethmuller, I., L.Bastiaans, J. Harbinson, C. Kempenaar y M. Kropff. 2006. Influence of the acetolactate synthase inhibitor Metsulfuron-,etyl on the operation, regulation and organization of photosynthesis in Solanum nigrum. Photosynth. Res. 88(1), 331-341. Doi: 10.1007/s11120-006-9062-z

Slapakauskas, V., E. Kazlauscas y S. Gliozeris. 2006. Effect of carboxylic acid hydrazid derivatives on the adventitious roots formation and photosynthetic electron transport in Phaseolus vulgaris. Sodininkysteirdarzininkyste 25(4), 305-315.

Slim, E., S. Shedeed, F. Asaad y A. El-Neklawy. 2012. Interactive effects of humic acid and water stress on chlorophyll and mineral nutrient contents of potato plants. J. Appl. Sci. Res. 8(1), 531-537.

Smith, A., S. Phatak y D. Emmatty. 1989. Metribuzin metabolism by tomato cultivars with low, medium, and high levels of tolerance to metribuzin. Pestic. Biochem. Physiol. 35(3), 284-290. Doi: 10.1016/0048-3575(89)90089-8

Steinhauser, M., D. Steinhauser, K. Koehl, F. Carrari, Y. Gibson, A. Fernie y M. Stitt. 2010. Enzyme activity profiles during fruit development in tomato cultivars and Solanum pennellii. Plant Physiol. 53(1), 80 98Doi: 10.1104/pp.110.154336

Taiz, L. y E. Zeiger. 2010. Plant physiology. $5^{\text {th }}$ ed. Sinauer Associates, Sunderland, MA.

Zhou, Q., W. Liu, Y. Zhang y K. Liu. 2007. Action mechanism of acetolactate synthase-inhibiting herbicide. Pestic. Biochem. Physiol. 89(1), 89-96. Doi: 10.1016/j.pestbp.2007.04.004 